\title{
ON FIBERING OF COBORDISM CLASSES
}

\author{
BY
}

R. E. STONG

ABSTRACT. This paper studies the problem posed by Conner and Floyd of finding which cobordism classes are represented by the total space of a fibering with prescribed base or fiber.

1. Introduction. The purpose of this note is to study the notion of fibering within a cobordism class introduced by Conner and Floyd [4]. These questions have also been studied by Conner [3], Burdick [2], and R. L. W. Brown [1], and to a certain extent this paper will provide some uniformity to their results.

Being given a cobordism class $a \in \Re_{m}$, the unoriented cobordism group, one says that $a$ fibers over $N^{n}$ with fiber $F^{m-n}$ if there is a differentiable fibering of closed manifolds

$$
\begin{array}{r}
F^{m-n} \rightarrow M^{m} \\
\downarrow \pi \\
N^{n}
\end{array}
$$

where $M^{m}$ belongs to the class $\alpha$.

There are two obvious ways to get new fiberings from a given fibering $F^{m-n}$ $\rightarrow M^{m} \stackrel{\pi}{\rightarrow} N^{n}:$

Construction 1. If there if a fibering $G^{n-q} \rightarrow N^{n} \stackrel{\pi^{\prime}}{\longrightarrow} Q^{q}$, the composite $\pi^{\prime} \circ$ $\pi: M^{m} \rightarrow Q^{q}$ is a fibering; and

Construction 2. If $f: Q^{q} \rightarrow N^{n}$ is a differentiable map, the induced (pullback) fibering is a fibering over $Q^{q}$.

The main result of the paper is the utter triviality:

Proposition 1.1. If $f: Q \rightarrow N^{n}$ and $f^{\prime}: Q^{\prime} \rightarrow N^{n}$ are differentiable maps representing the same class in the bordism of $N$, then the total spaces of the induced fiberings over $Q$ and $Q^{\prime}$ are bordant.

Proof. Let $F: V \rightarrow N$ be a differentiable map with boundary $f \cup f^{\prime}$. Then the total space of the induced bundle over $V$ has boundary the disjoint union of the total spaces of the induced fiberings over $Q$ and $Q^{\prime}$.

Having proved our main result, the remainder of this note is devoted to applications.

Received by the editors May 31, 1972.

AMS (MOS) subject classifications (1970). Primary 57D90. 
The author is indebted to the National Science Foundation for financial support during this work.

\section{Burdick's theorem.}

Proposition 2.1. If $a \in \Re_{m}$ is fibered over $N^{n}$ and $f: N^{\prime} \rightarrow N$ is bordant to the identity map of $N$, then $a$ is fibered over $N^{\prime}$.

Proof. $f: N^{\prime} \rightarrow N$ and $1: N \rightarrow N$ are bordant, and the induced fibering via 1 represents $a$.

For any manifold $\left(N^{\prime}\right)^{n}$ which bounds, there is a degree one map $f:\left(N^{\prime}\right)^{n} \rightarrow$ $S^{n}$, the $n$-sphere, and any such map is bordant to the identity map of $S^{n}$. One explicit construction for such a map may be obtained by letting $f^{\prime}: S^{n} \cup N^{\prime} \rightarrow S^{n}$ be the identity on $S^{n}$ and the point map $f^{\prime}\left(N^{\prime}\right)=p$, which is bordant to the identity since $N^{\prime}$ bounds, then doing surgery on an imbedded $S^{0}$, given by $p$ and a point of $N^{\prime}$ (see [7]), to cobord this to a map of the connected sum $S^{n} \# N^{\prime}$, which is just $N^{\prime}$, into $S^{n}$. This gives

Corollary 2.2. If $a \in \Re_{m}$ is fibered over $S^{n}$ and $\left(N^{\prime}\right)^{n}$ is any bounding $n$ manifold, then a fibers over $\left(N^{\prime}\right)^{n}$.

For any manifold $Q^{q}$ of dimension $q \leq n$, there is a bounding manifold of dimension $n, Q^{q} \times S^{n-q}$, fibered over $Q^{q}$, and applying Construction 1 gives

Proposition 2.3. If $a \in \Re_{m}$ is fibered over $S^{n}$ then for any manifold $Q^{q}$ of dimension $q \leq n$, a fibers over $Q^{q}$.

Note. For $Q^{q}=S^{1}$ this is Burdick's theorem (1.2). Charles Giffen once pointed out to me that Burdick's result followed by constructing a cobordism of $S^{1} \times S^{n-1}$ to $S^{n}$ which retracts onto $S^{n}$ and pulling back the fibering, just as was done here.

Proposition 2.4. If $a \in \Re_{m}$ is fibered over $N^{n}$ with $m>n$ and $f: N^{\prime} \rightarrow N$ is a degree-one map with $\beta \in \mathcal{N}_{m}^{m}$ the class of the induced fibration, then $\alpha \equiv \beta$ modulo decomposables in $\Re_{m}$.

Proof. Since $f$ has degree one, $f \cup 1$ is decomposable in the bordism of $N$, so there exist manifolds $Q_{i}^{j}, j<n$, and $P_{i}^{n-j}$ and maps $g_{j}^{i}: Q_{i}^{j} \rightarrow N$ with $1: N \rightarrow$ $N$ bordant to the sum of $f$ and the maps $g_{j}^{i}{ }^{\circ} \mathrm{pr} Q: P_{i}^{n-j} \times Q_{i}^{j} \rightarrow N$ where $\mathrm{pr}_{Q}$ is the projection on $Q_{i}^{j}$. If $\gamma_{i}^{m+j-n} \in \Re_{m+j-n}$ is the class of the fibration induced over $Q_{i}^{j}$ via $g_{j}^{i}$, then $\alpha=\beta+\Sigma_{i, j}\left[P_{i}^{n-j}\right] \gamma_{i}^{m+j-n}$, with $n-j>0$ and $m+j-n$ $>0$.

3. Fiberings over projective spaces. For a vector bundle $\xi$ over a space $X$, let $R P(\xi)$ be the projective space bundle of $\xi$ consisting of lines in the fibers 
of $\xi$ and let $\lambda$ be the canonical line bundle over $R P(\xi)$ with total space the pairs $(\alpha, x)$, with $\alpha$ a line in a fiber of $\xi$ and $x$ a vector of $\xi$ in the line $\alpha$. Denote by $\underline{n}$ the trivial $n$-plane bundle over a space, and let $R P(n)=R P(\underline{n+1})$, where $\underline{n+1}$ is the trivial bundle over a point.

Lemma 3.1. If $\lambda$ is the canonical line bundle over $R P(n)$, then $R P(\lambda \oplus \underline{1})$ is the connected sum $R P(n+1) \# R P(n+1)$.

Proof. $R P(\lambda \oplus \underline{1})$ is the quotient of $S^{n} \times S^{1},\left\{\left(\left(x_{1}, \ldots, x_{n+1}\right),\left(y_{1}, y_{2}\right)\right) \in\right.$ $\left.R^{n+1} \times R^{2} \mid\|x\|=\|y\|=1\right\}$, obtained by identifying $\left(x,\left(y_{1}, y_{2}\right)\right)$ and $\left(-x,\left(-y_{1}, y_{2}\right)\right)$ to get the sphere bundle $S(\lambda \oplus \underline{1})$ and identifying $\left(x,\left(y_{1}, y_{2}\right)\right)$ and $\left(x,\left(-y_{1},-y_{2}\right)\right)$ to get the projective space bundle. The sets $\left\{\left.\left(x,\left(y_{1}, y_{2}\right)\right)|| y_{1}\right|^{2} \leq 1 / 2\right\}$ and $\{(x$, $\left.\left.\left(y_{1}, y_{2}\right)\right)\left.|| y_{2}\right|^{2} \leq 1 / 2\right\}$ split $R P(\lambda \oplus \underline{1})$ into two copies of the disc bundle $D(\lambda)$ joined along their boundary $S^{n}$. Since $R P(n+1)$ is $D(\lambda)$ with an $(n+1)$-disc attached along the boundary, this exhibits the splitting as a connected sum.

Now let $\tilde{\lambda}$ be the $n$-plane bundle consisting of pairs $(\alpha, y)$ with $\alpha$ a line in $R^{n+1}$ and $y$ a vector orthogonal to the line $\alpha$, so that $\tilde{\lambda}$ is the complement of $\lambda$ over $R P(n)$. Let $i: R P(\lambda \oplus \underline{1}) \hookrightarrow R P(\lambda \oplus \tilde{\lambda} \oplus \underline{1})$ be the inclusion, and let $\pi$ : $R P(\lambda \oplus \tilde{\lambda} \oplus \underline{1})=R P(n) \times R P(n+1) \rightarrow R P(n+1)$ be the projection of the trivial bundle onto its fiber. Then $\pi \circ i: R P(\lambda \oplus \underline{1}) \rightarrow R P(n+1)$ is the standard map which classifies the canonical bundle over $R P(\lambda \oplus \underline{1})$.

Lemma 3.2. The map $\pi \circ i: R P(\lambda \oplus \underline{1}) \rightarrow R P(n+1)$ is identifiable with the map $R P(n+1) \# R P(n+1) \rightarrow R P(n+1)$ which collapses one summand to a point.

Proof. The map $\pi \circ i: R P(\lambda \oplus \underline{1}) \rightarrow R P(n+1)$ sends the line consisting of multiples of $((\alpha, x), t) \in E(\lambda) \times R$, where $E(\lambda)$ is the total space of $\lambda$, into the line of $R^{n+2}=R^{n+1} \times R$ consisting of multiples of $(x, t)$. The subspace $R P(\underline{1})(x=0)$ is sent into the point $R P(0)$ of $R P(n+1)$ given by the line of multiples of $(0,1)$ and the complement of $R P(\underline{1})$ maps homeomorphically to $R P(n+1)-R P(0)$, for $(x, t)$ with $x \neq 0$ determines the line $\alpha$ as the multiples of $x$. Thus $\pi \circ i$ is the map obtained by collapsing $R P(\underline{1})$ to a point. Since the decomposition of $R P(\lambda \oplus \underline{1})$ into $R P(n+1) \# R P(n+1)$ was given by decomposing into two copies of $D(\lambda)$ which were the tubular neighborhoods of $R P(\lambda)$ and $R P(\underline{1})$, this is just the collapse onto one summand.

Lemma 3.3. The map $f: R P(\lambda \oplus \underline{1}) \cup R P(n+1) \rightarrow R P(n+1)$ given by $\pi \circ i$ on $R P(\lambda \oplus \underline{1})$ and by a point map to $R P(0)=p$ on $R P(n+1)$ is bordant to the identity.

Proof. The map $(1 \cup p \cup p): 3 R P(n+1) \rightarrow R P(n+1)$ is bordant to the identity and $f$ is obtained by surgery along an imbedded $S^{0}$.

I must now apologize for having to perform a brutal computation, but at some stage one must get his hands filthy. 
Lemma 3.4. Let $R P\left(n_{1}, n_{2}, \cdots, n_{k}\right)$ be the projective space bundle of the bundle $\lambda_{1} \oplus \cdots \oplus \lambda_{k}$ over $R P\left(n_{1}\right) \times \cdots \times R P\left(n_{k}\right)$, where $\lambda_{i}$ is the pullback of the canonical bundle over the ith factor. Then for $k>1, R P\left(n_{1}, \ldots, n_{k}\right)$ is indecomposable in $\Re_{*}$ if and only if

$$
\left(\begin{array}{c}
n+k-2 \\
n_{1}
\end{array}\right)+\cdots+\left(\begin{array}{c}
n+k-2 \\
n_{k}
\end{array}\right)
$$

is odd, where $n=n_{1}+\cdots+n_{k}$. (Note: The manifold has dimension $\left.n+k-1.\right)$

Proof. The cohomology with $Z_{2}$ coefficients of $X=R P\left(n_{1}, n_{2}, \cdots, n_{k}\right)$ is the free module over the cohomology of $Y=R P\left(n_{1}\right) \times \cdots \times R P\left(n_{k}\right)$ on $1, c, \cdots$, $c^{k-1}$ where $c$ is the first Stiefel-Whitney class of the canonical line bundle over $X$, with the relation

$$
c^{k}=w_{1} c^{k-1}+\cdots+w_{r} c^{k-r}+\cdots+w_{n}
$$

where $w_{i}$ is the $i$ th Stiefel-Whitney class of $\lambda_{1} \oplus \cdots \oplus \lambda_{k}$. Thus, inductively, $c^{k-1+i}=\bar{w}_{i} c^{k-1}+$ terms with lower powers of $c$, where $\bar{w}_{i}$ is the $i$ th dual StiefelWhitney class of $\lambda_{1} \oplus \cdots \oplus \lambda_{k}$ (see [3, Lemma (3.1)]). Letting $\alpha_{i}$ be the pullback of the nonzero class in $H^{1}\left(R P\left(n_{i}\right) ; Z_{2}\right)$, the Stiefel-Whitney class of $X$ is $\Pi_{i=1}^{k}\left(1+a_{i}\right)^{n_{i}+1} \cdot \Pi_{i=1}^{k}\left(1+c+a_{i}\right)$, and so the $s$-class of $X$ is

$$
\begin{aligned}
s_{n+k-1} & =\sum_{i}\left(n_{i}+1\right) \alpha_{i}^{n+k-1}+\sum_{i}\left(c+\alpha_{i}\right)^{n+k-1} \\
& =k c^{n+k-1}+\sum_{j=1}^{n}\left(\begin{array}{c}
n+k-1 \\
j
\end{array}\right) s_{j} c^{n+k-1-j} \\
& =c^{k-1}\left[k \bar{w}_{n}+\sum_{j=1}^{n}\left(\begin{array}{c}
n+k-1 \\
j
\end{array}\right) s_{j} \bar{w}_{n-j}\right]
\end{aligned}
$$

where $s_{j}=\sum_{i=1}^{k} \alpha_{i}^{j}$ is the $j$ th $s$-class of $\lambda_{1} \oplus \cdots \oplus \lambda_{k}$ (assuming $n+k-1>n_{i}$ for each $i$ or that $k>1$ ) as in [3, Theorem (4.1)]. The value of this characteristic class on $X$ is the coefficient of $x_{1}^{n_{1}} \cdots x_{k}^{n_{k}}$ in

$$
\begin{aligned}
& \frac{\left(1+x_{1}\right)^{n+k-1}+\cdots+\left(1+x_{k}\right)^{n+k-1}}{\left(1+x_{1}\right) \cdots\left(1+x_{k}\right)} \\
& \quad=\frac{\left(1+x_{1}\right)^{n+k-2}}{\left(1+x_{2}\right) \cdots\left(1+x_{k}\right)}+\cdots+\frac{\left(1+x_{k}\right)^{n+k-2}}{\left(1+x_{1}\right) \cdots\left(1+x_{k-1}\right)}
\end{aligned}
$$

which is

$$
\left(\begin{array}{c}
n+k-2 \\
n_{1}
\end{array}\right)+\cdots+\left(\begin{array}{c}
n+k-2 \\
n_{k}
\end{array}\right)
$$


Corollary 3.5. There exist indecomposable manifolds $M^{m}$ fibered over $R P(n)$ with

(a) $m=2 s \geq 4, n=2 s-2$,

(b) $m=2^{p}(2 q+1)-1, p, q>0, n=2^{p+1} q-2$.

Proof. Let $M^{m}=R P(n, 0, \ldots, 0)$ with $m-n$ zeros, noting that $\left(\begin{array}{c}2 s-1 \\ 2 s-2\end{array}\right)+$ $2\left(\begin{array}{c}2 s-1 \\ 0\end{array}\right) \equiv 1(\bmod 2)$ and $\left(\begin{array}{c}2 p+1 \\ 2 p+2 \\ 2 p-1 \\ p-2\end{array}\right)+\left(2^{p}+1\right)\left(\begin{array}{c}2 p+1 \\ 0+2 p-2\end{array}\right) \equiv 1(\bmod 2)$.

Note. See Theorems (5.3) and (5.4) of [4].

Question. Are these the largest values of $n$ for which there is an indecomposable $M^{m}$ fibered over $R P(n)$ with $m>n$ ? For $m$ even, this is true, since for $M^{m}$ fibered over $N^{m-1}, M^{m}$ is an $S^{1}$ bundle over a finite cover of $N^{m-1}$, and any $S^{1}$ bundle bounds. For $m=5$, any $M^{5}$ fibered over $R P(3)$ has tangent bundle stably the bundle along the fibers which is a 2-plane bundle, and so $w_{2} w_{3}[M]=0$ and $M$ bounds. (Note. This answers the question of [4, p. 41], although Brown answered it better by showing that no indecomposable five manifold fibers over $S^{2}$, as was asked in [3, p. 303].)

4. The theorems of Conner and Floyd.

Proposition 4.1. There are indecomposable classes $a \in \Re_{m}$ fibered over $R P(j)$ for all $j \leq n$ where

(a) $m=2 s \geq 4, n=2 s-2$, and

(b) $m=2^{p}(2 q+1)-1, p, q>0, n=2^{p+1} q-2$.

Proof. Such classes exist for $j=n$, and if such an indecomposable exists for $R P(k)$, then $f: R P(\lambda \oplus \underline{1}) \cup R P(k) \rightarrow R P(k)$, as in Lemma 3.3, gives an indecomposable fibered over $R P(\lambda \oplus \underline{1})$ and hence over $R P(k-1)$.

Note. The classes $a \in \Re_{m}$ fibered over $R P(j)$ with $j \leq n$ given above may be written down explicitly. They are given by the iterated projective space bundles

$$
R P(j) \leftarrow R P(\lambda \oplus \underline{1}) \leftarrow \cdots \leftarrow R P(\lambda \oplus \underline{1}) \leftarrow R P(\lambda \oplus \underline{(m-n)})
$$

where each $\lambda$ is the canonical bundle of the preceding projective space bundle, and $R P(\lambda \oplus \underline{1})$ occurs $(n-j)$ times. In particular, the fiberings all have structure group $Z_{2}$.

This gives Theorems (5.3) and (5.4) of [4]. In fact, these are exactly the same representatives given by Conner and Floyd.

Corollary 4.2. Every positive-dimensional cobordism class contains a representative fibered over $R P(2)$.

Proof. Proposition 4.1 exhibits indecomposables with this property in every dimension not of the form $2^{s}-1$ except 2 , and $R P(2)$ is indecomposable.

Note. This is Corollary (5.5) of [4]. 
Corollary 4.3. Every positive-dimensional cobordism class is of the form $a+$ $n[R P(2)]^{s}$ where $n=0,1$, and a fibers over the Klein bottle $K=R P(2) \# R P(2)$.

Proof. If $\beta \in \Re_{m}, m>0, \beta$ fibers over $R P(2)$ with fiber $F$ and pulling back over $f: K \cup R P(2) \rightarrow R P(2), \beta+[F] \cdot[R P(2)]$ fibers over $K$. By induction on $m$, the result holds for $[F]$.

Proposition 4.4. The following sets of cobordism classes are the same:

$S(a)$ : Those classes for which the mod 2 Euler characteristic is zero.

$S(\mathrm{~b})$ : Those classes which fiber over $S^{1}$.

$S(c)$ : Those classes which fiber over the Klein bottle.

Proof. Since $K$ fibers over $S^{1}$ and any fibering over $S^{1}$ has zero mod 2 Euler characteristic, $S(\mathrm{c}) \subset S(\mathrm{~b}) \subset S(\mathrm{a})$. Since $[R P(2)]^{s}$ has odd Euler characteristic, Corollary 4.3 gives $S(\mathrm{c})=S(\mathrm{a})$.

Note. Each of these is the ideal generated by the classes $a$ of Proposition 4.1 with $j=1$, so the fiberings may be taken to have structure group $Z_{2}$. This gives Theorem (4.5) of [4], and in fact these are the same representatives given in the proof of Theorem (4.3). The generators there have the form $V(n, k), k \geq 2$, and hence fiber over the Klein bottle $V(0,2)$ using a point as 0 -manifold.

Remark. One may use exactly the same constructions with complex and quaternionic projective space bundles, and apply the doubling isomorphisms of cohomology or the existence of conjugations and automorphisms of $H$ over $C$ to construct involutions to show:

If $a \in \eta_{m}$ is an indecomposable fibered over $R P(j)$ as constructed above, then $a^{2}$ fibers over $C P(j)$ and $\alpha^{4}$ fibers over $H P(j)$.

5. Brown's fiberings. In this section, fiberings over $S^{2}$ will be constructed to generate the ideal of classes which fiber over $S^{2}$.

Proposition 5.1. There exist indecomposable manifolds $M^{2 s}$ fibered over $C P(j)$ for $j<s$.

Proof. Let $\eta$ be the canonical complex line bundle over $C P(s-1)$ and $M^{2 s}$ $=R P(\eta \oplus \underline{1})$. If $g: R P(2 s-2) \rightarrow C P(s-1)$ with $g^{*}(\eta)=\lambda \otimes C=\lambda \oplus \lambda, g$ is degree one, so $[R P(\eta \oplus \underline{1})] \equiv[R P(\lambda \oplus \lambda \oplus \underline{1})] \bmod$ decomposables. Now $R P(\lambda \oplus \lambda \oplus \underline{1})=$ $R P(\underline{1} \oplus \underline{1} \oplus \lambda)$, for $R P(\rho \otimes \xi)=R P(\xi)$ if $\rho$ is a line bundle since tensoring with a nonzero vector in $\rho$ establishes a one-to-one correspondence between lines in a fiber of $\xi$ and lines in a fiber of $\rho \otimes \xi$. Since $R P(\lambda \oplus \underline{2})$ is indecomposable, so is $M$. Applying the degree one map $C P(\eta \oplus(\underline{1} \otimes C)) \rightarrow C P(k+1)$ pulls indecomposables down to $C P(k)$, and the result follows.

Proposition 5.2 (Brown [1]). Every positive-dimensional class in $\Re_{*}$ bas the form $\alpha+n x_{2}^{i} x_{5}^{\epsilon}$, with $n, \epsilon=0,1$, with a fibered over $S^{2}, x_{j} \in \Re_{j}$ being an indecomposable. 
Proof. By Proposition 4.1 there are indecomposables fibered over $R P(3)$, and hence over $C P(1)=S^{2}$ except in dimensions 2, 4 and 5. By Proposition 5.1 there is an indecomposable $M^{4}$ fibered over $S^{2}$, and by the remark in $\$ 4$ the square of the nonzero class in $\eta_{5}$ fibers over $S^{2}$.

Brown proved also that $x_{2}^{i} x_{5}^{\epsilon}$ cannot fiber over $S^{2}$, and hence we have exhibited generators for the ideal of classes which fiber over $S^{2}$.

Proposition 5.3. Every positive-dimensional cobordism class bas the form a $+\beta$ where a fibers over $C P(2)$ and $\beta$ is either zero or

$$
x_{2}, \quad x_{4}, x_{5}, x_{2} x_{4}, x_{2} x_{5}, x_{4} x_{5}, \text { or } x_{2} x_{4} x_{5}
$$

and these classes do not fiber over CP(2).

Proof. By the remark in $\$ 4$, every square fibers over $C P(2)$. By Proposition 5.1 , there are indecomposables $M^{2 s}$ fibered over $C P(2)$ for $2 s \geq 6$, and by Proposition 4.1 there are indecomposables $M^{2 p(2 q+1)-1}$ fibered over $R P(5)$ and hence $C P(2)$ if $2^{p+1} q-2 \geq 6$ or $2^{p} q \geq 4$, i.e. except in dimension 5 .

To complete the proof one need only exhibit a class $\beta \in \Re_{11}$ which does not fiber over $C P(2)$. If $\pi: M^{11} \rightarrow C P(2)$ is a fibering, let $N^{9}=\pi^{-1}(C P(1))$ be the induced fibering over $C P(1)$ and $F^{7}=\pi^{-1}(C P(0))$ the fiber, with $i: N \rightarrow M, j$ : $F \rightarrow N$ the inclusions. Then $F$ has trivial normal bundle in $M$, so $j^{*} i^{*} v_{4}(M)=$ $v_{4}(F)=0$ for the $\mathbb{W u}$ class $v_{4}$ is zero in a 7 -manifold. Thus $i^{*} v_{4}(M)$ is in the image of $H^{4}(N, F) \cong H^{2}(F) \otimes H^{2}\left(D^{2}, S^{1}\right)$ so $i^{*} \mathrm{Sq}^{3} v_{4}(M)=\mathrm{Sq}^{3} i^{*} v_{4}(M)$ is zero. Thus $\mathrm{Sq}^{3} v_{4}(M)$ is in the image of $H^{7}(M, N) \cong H^{3}(F) \otimes H^{4}\left(D^{4}, S^{3}\right)$ and so $\mathrm{Sq}^{4} \mathrm{Sq}^{3} v_{4}(M)=0$. Thus the characteristic number $v_{4} \mathrm{Sq}^{3} v_{4}[M]=\mathrm{Sq}^{4} \mathrm{Sq}^{3} v_{4}[M]$ is zero. By direct computation one sees that $v_{4} \mathrm{Sq}^{3} v_{4}\left[R P(2) \times R P(4) \times M^{5}\right] \neq 0$ if $M^{5}$ does not bound, giving the result.

Proposition 5.4. Every positive-dimensional cobordism class bas the form $\alpha+\beta$ where $\alpha$ fibers over $R P(2) \times R P(2)$ and $\beta$ is either zero or $x_{2}, x_{4}, x_{5}$ and these classes do not fiber over $R P(2) \times R P(2)$.

Proof. Every positive-dimensional class fibers over $R P(2)$, so every decomposable class fibers over $R P(2) \times R P(2)$. There exist indecomposables fibered over $R P(6)$, hence over $R P(2) \times R P(4)$ and $R P(2) \times R P(2)$, in dimensions larger than 7, using a degree one map $R P(2) \times R P(4) \rightarrow R P(6)$. Then $R P(2,2,0)$ is an indecomposable 6 manifold fibered over $R P(2) \times R P(2)$, and so the only indecomposables which do not fiber are in dimensions 2,4 , and 5 . In these dimensions a fibering over $R P(2) \times R P(2)$ has empty, finite, or 1 -dimensional fiber, so represents zero or a multiple of $R P(2) \times R P(2)$.

6. Fiberings over two-manifolds. In this section, we will determine explicitly which cobordism classes fiber over each 2-dimensional manifold. There are the following sets of classes: 
$\Sigma_{1}$ : All positive dimensional classes.

$\Sigma_{2}$ : All classes with mod 2 Euler characteristic zero.

$\Sigma_{3}$ : Those classes $\alpha \in \Re_{m}$ for which all numbers divisible by $w_{m}$ and $w_{m-2}$ (for $m$ odd) are zero (i.e. Brown's class).

Proposition 6.1. If $\mathrm{M}^{2}$ is a compact, connected 2-manifold, then the set of classes in $\Re_{*}$ which fiber over $M^{2}$ is

(a) $\Sigma_{1}$ if $M^{2}$ is the connected sum of an odd number of projective planes,

(b) $\Sigma_{2}$ if $M^{2}$ is the connected sum of an even number of projective planes or a connected sum of tori, and

(c) $\Sigma_{3}$ if $M^{2}$ is the sphere.

Note. According to Massey [6, Theorem 5.1], every compact connected 2-manifold is of one of these types, making the result complete.

Proof. If $M^{2}$ is the connected sum of an odd number of projective planes, collapsing onto one summand is bordant to the identity of $R P(2)$, giving part (a). If $M^{2}$ is the connected sum of an even number of projective planes or a sum of tori, the mod 2 Euler characteristic of $M$ and any fibering over $M$ is zero. If $M$ is the connected sum of an even number of projective planes, collapsing onto two summands is bordant to the identity map of the Klein bottle, for which the set of classes is $\Sigma_{2}$. If $M$ is the connected sum of tori, collapsing onto one summand is bordant to the identity map on $S^{1} \times S^{1}$. By Lemma 3.4, $R P(2,1,0)$ is an indecomposable 5-manifold fibered over $R P(2) \times R P(1)$ which pulls back to $K \times R P(1)$ which fibers over $S^{1} \times S^{1}$. Since adjoining $x_{5}$ to $\Sigma_{3}$, those classes fibered over $S^{2}$, gives $\Sigma_{2}$, the classes fibered over $S^{1} \times S^{1}$ are precisely those of $\Sigma_{2}$.

If $M^{2}$ is any 2-manifold, i.e. not necessarily connected, this gives the general case quite easily.

Case I. If the fibers over distinct components of $M$ are allowed to vary, then the set of classes which fiber over $M$ is the largest of the sets fibering over the components of $M$, with $\Sigma_{1} \supset \Sigma_{2} \supset \Sigma_{3}$.

Case II. If the fiber is required to be the same over all components of $M$, then the set of classes fibered over $M$ is

(a) $\Sigma_{3}$ if $M$ is a union of spheres,

(b) $\Sigma_{1}$ if $M$ does not bound,

(c) $\Sigma_{2}$ in all other cases.

7. Fibering over products of circles. A cursory glance at the results obtained so far indicate that it is much easier to fiber manifolds over products of circles than over spheres. Largely as a curiosity, one has $\left(S^{1}\right)^{k}$ for

Proposition 7.1. There exist indecomposable manifolds $M^{m}$ fibered over 
(a) $m=4 s, 4 s+2$ with $s \geq 1$ and $k=2 s$,

(b) $m=2^{p}(2 q+1)-1$ with $p, q>0$ and $k=2^{p} q$.

Proof. For $M^{4 s}$ take

and for $M^{4 s+2}$ take

$$
R P(\underbrace{1, \ldots, 1}_{2 s}, 0)
$$

$$
R P(\underbrace{1, \ldots, 1}_{2 s}, 0,0,0) .
$$

For $M^{5}$, we have constructed a fibering over $S^{1} \times S^{1}$, while for $M^{2 p(2 q+1)-1}$ with $2^{p} q \geq 3$, let $s+3=2^{p} q$ and form the indecomposable

$$
R F(2^{p}, 3, \underbrace{1, \ldots, 1}_{s}, 0) \text {. }
$$

This pulls back to $R P\left(2^{p}\right) \times R P(2) \times R P(1) \times(R P(1))^{s}$ by a degree one map of $R P(2) \times$ $R P(1)$ into $R P(3)$, and then use degree one maps of fiberings over $R P(1)$ into $R P\left(2^{p}\right)$ and $R P(2)$ to obtain a fibering over $(R P(1))^{s+3}$.

On the grounds that every paper should contain at least one result which the reader cannot prove by inspection, let us now prove

Proposition 7.2. The set of cobordism classes $a \in \Re_{m}$ which fiber over $\left(S^{1}\right)^{4}$ is precisely the set of classes for which all Stiefel-Whitney numbers divisible by $w_{m}, w_{m-1}, w_{m-2}$, and $w_{m-3}$ are zero.

Proof. If $M^{m}$ fibers over $\left(S^{1}\right)^{4}$, then the tangent bundle of $M^{m}$ is the pull back of the tangent bundle of $\left(S^{1}\right)^{4}$, which is trivial, and the bundle along the fibers which is an $(m-4)$-plane bundle, so $w_{m}=w_{m-1}=w_{m-2}=w_{m-3}=0$.

By Proposition 7.1, there are indecomposable manifolds in every dimension greater than 6 which fiber over $\left(S^{1}\right)^{4}$, and letting $x_{4}, x_{5}, x_{6}$ be the classes of indecomposable manifolds which fiber over $\left(S^{1}\right)^{2}$, the ideal of manifolds fibered over $\left(S^{1}\right)^{4}$ contains the ideal generated by $x_{4}^{2}, x_{5}^{2}, x_{6}^{2}, x_{4} x_{5}, x_{4} x_{6}, x_{5} x_{6}$ and $x_{i}$ for all $i>6$. To complete the theorem one then need only show that the classes $x_{2}^{i} x_{5}$, and $x_{2}^{j}, x_{2}^{j-2} x_{4}, x_{2}^{j-3} x_{6}$ are distinguished by characteristic numbers involving $w_{m}, w_{m-1}, w_{m-2}$, and $w_{m-3}$.

For $x_{2}^{i} x_{5}$, Brown has shown that $w_{2} w_{2 i+3}\left[x_{2}^{i} x_{5}\right]$ is nonzero and so one need only consider the even dimensional case, and so one considers the classes $x_{2}^{j}$, $x_{2}^{j-2} x_{4}, x_{2}^{j-3} x_{6}$ of dimension $2 j$. Now the partitions $s_{\omega}$ for

$$
\omega=(\underbrace{1, \ldots, 1}_{2 j}),(\underbrace{1, \ldots, 1}_{2 j-4}, 2,2) \text {, and }(\underbrace{1, \ldots, 1}_{2 j-6}, 2,2,2)
$$

each contain at least $2 j-3$ entries, so vanish for $2 j-4$ plane bundles and hence lie in the ideal generated by $w_{2 j}, w_{2 j-1}, w_{2 j-2}$, and $w_{2 j-3}$. 
In order to show that these classes distinguish the given bordism elements, let $\theta: \Re_{*} \rightarrow Z_{2}\left[t_{1}, t_{2}, t_{3}, \ldots\right]$ be the ring homomorphism defined by $\theta([M])=$ $\Sigma_{\omega} s_{\omega}[M] \cdot t_{\omega}$ where $t_{\left(i_{1}, \cdots, i_{r}\right)}=t_{i_{1}} \cdots t_{i_{r}}$, and reduce modulo the ideal generated by the $t_{i}$ for $i>2$, to give a ring homomorphism $\theta^{\prime}: \Re_{*} \rightarrow Z_{2}\left[t_{1}, t_{2}\right]$ given by

$$
\theta^{\prime}\left(\left[M^{m}\right]\right)=\sum_{p+2 q=m} s(\underbrace{1, \cdots, 1}_{p}, \underbrace{2, \cdots, 2}_{q})[M] t_{1}^{p} t_{2}^{q} .
$$

We then claim $\theta^{\prime}([R P(2)])=t_{1}^{2}+t_{2}, \theta^{\prime}([R P(1,1,0)])=t_{2}^{2}$ and $\theta^{\prime}([R P(1,1,0,0,0)])$ $=t_{1}^{2} t_{2}^{2}$, so that the characteristic numbers are given by

\begin{tabular}{c|c|c|c}
$s_{\omega}([M])$ & $R P(2)^{j}$ & $R P(2)^{j-2} R P(1,1,0)$ & $R P(2)^{j-3} R P(1,1,0,0,0)$ \\
\hline$s(\underbrace{1, \ldots, 1}_{2 j})$ & 1 & 0 & 0 \\
$s(\underbrace{1, \ldots, 1,2,2}_{2 j-4})$ & $\left(\begin{array}{l}j \\
2\end{array}\right)$ & 1 & 1 \\
$s(\underbrace{1, \ldots, 1,2,2,2}_{2 j-6})$ & $\left(\begin{array}{l}j \\
3\end{array}\right)$ & $\left(\begin{array}{c}j-2 \\
1\end{array}\right)$ & $\left(\begin{array}{c}j-3 \\
1\end{array}\right)$
\end{tabular}

and since this matrix is nonsingular $(j-2 \not \equiv j-3 \bmod 2)$, these classes distinguish $x_{2}^{j}, x_{2}^{j-2} x_{4}$, and $x_{2}^{j-3} x_{6}$.

To compute $\theta^{\prime}$ on these classes, we compute only $\theta^{\prime}([R P(1,1,0,0,0])$. In the notation of Lemma 3.4, the Stiefel-Whitney class of $X=R P(1,1,0,0,0)$ is $\left(1+c+\alpha_{1}\right)\left(1+c+\alpha_{2}\right)(1+c)^{3}$ with the relations $\alpha_{i}^{2}=0, c^{3}\left(c+\alpha_{1}\right)\left(c+\alpha_{2}\right)=0$ so that $c^{6}[X]=1, c^{5}\left(\alpha_{1}+\alpha_{2}\right)[X]=0$, and $c^{4} \alpha_{1} \alpha_{2}[X]=1$. Since $\theta^{\prime}$ behaves as a ring homomorphism in the cohomology characteristic classes, $\theta^{\prime}([X])$ is the value of the 6-dimensional component of

$$
\left(1+\left(c+a_{1}\right) t_{1}+c^{2} t_{2}\right) \cdot\left(1+\left(c+a_{2}\right) t_{1}+c^{2} t_{2}\right) \cdot\left(1+c t_{1}+c^{2} t_{2}\right)^{3}
$$

on the fundamental class of $X$. We leave it to the reader to calculate this mess, and analyze $R P(1,1,0)$ and $R P(2)$.

It is very tempting to conjecture that the set of classes $a \in \Re_{m}$ fibered over $\left(S^{1}\right)^{k}$ is precisely the set of classes for which all numbers divisible by $w_{m}$, $w_{m-1}, \cdots, w_{m-k+1}$ are zero. This is true if $k=1,2$ or 4 , and is true for $k=3$ if there is an indecomposable 6 manifold fibered over $\left(S^{1}\right)^{3}$ (which must be in the class of $R P(1,1,0,0,0)+R P(1,1,0) \cdot R P(2))$.

8. Classes with a given fiber. Although the question has not been previously mentioned, it seems interesting to ask: Given a closed manifold $F^{k}$, which classes $a \in \Re_{m}$ are represented by a fibering with fiber $F$ ? The set of classes which can be so represented forms an ideal in $\Re_{*}$.

Our first result is 
Proposition 8.1. Every positive-dimensional class in $\Re_{*}$ is represented by a fibering with fiber $R P(2)$.

Proof. Let $\sigma: \Re_{m}\left(\mathrm{BO}_{3}\right) \rightarrow \Re_{m+2}$ be the homomorphism assigning to the $3-$ plane bundle $\xi$ over $M^{m}$ the bordism class of $R P(\xi)$ (see $[3, \S 2]$ ). As in Lemma (2.2) of [3], 跛 $\left(\mathrm{BO}_{3}\right)$ has a base over $\Re_{*}$ consisting of products $R P\left(n_{1}\right) \times R P\left(n_{2}\right)$ $\times R P\left(n_{3}\right), n_{1} \leq n_{2} \leq n_{3}$, with the bundle $\lambda_{1} \oplus \lambda_{2} \oplus \lambda_{3}$, and $\sigma$ is an $\Re_{*}$ module homomorphism. Thus, the result will be obtained, and $\sigma$ will be proved epic, by exhibiting indecomposable manifolds $R P\left(n_{1}, n_{2}, n_{3}\right)$ in every dimension.

For $M^{2 s}$, take $R P(2 s-2,0,0)$, as in Corollary 3.5 for $s \geq 2$ and which is $R P(2)$ for $s=1$. For $M^{n}, n=2^{p}(2 q+1)-1$ with $p>0, q>0$, take $R P\left(2^{p}, 2^{p+1} q-3,0\right)$.

We now wish to describe those classes which fiber with fiber $R P(3)$, beginning with a result interesting in its own right.

Proposition 8.2. If $a \in \Re_{n}$ is represented by a fibering with fiber $R P(2 k+1)$, then $w_{n}(\alpha)=w_{1}^{n}(\alpha)=0$.

Proof. Let $\pi: M^{n} \rightarrow Q^{n-2 k-1}$ be a fibering with fiber $R P(2 k+1)$ representing a. Then $w_{n}(\alpha)=w_{n}[M]$ is the mod 2 Euler characteristic $\chi(M)=$ $\chi(R P(2 k+1)) \cdot \chi(Q)=0$. Letting $i: R P(2 k+1) \rightarrow M$ be the inclusion of a fiber, $i$ is an imbedding with trivial normal bundle, so $i^{*}\left(w_{1}(M)\right)=w_{1}(R P(2 k+1))=0$, and from the Serre spectral sequence $w_{1}(M) \in$ image $\pi^{*}$, so $w_{1}(M)=\pi^{*}(a)$ for some $a \in H^{1}\left(Q ; Z_{2}\right)$. Thus $w_{1}(M)^{n-2 k}=\pi^{*}\left(a^{n-2 k}\right)=0$, for $H^{n-2 k}\left(Q ; Z_{2}\right)=0$, and so $w_{1}^{n}[M]=0$.

Proposition 8.3. The class $a \in \Re_{n}$ is represented by a fibering with fiber. $R P(3)$ if and only if $w_{n}(\alpha)=w_{1}^{n}(\alpha)=0$.

Proof. If $a$ fibers with fiber $R P(3)$, then $w_{n}(\alpha)=w_{1}^{n}(\alpha)=0$ by Proposition 8.2.

If $m=2^{p}(2 q+1)-1, p, q>0$, let $M^{m}=R P\left(2^{p}, 2^{p} q-1,2^{p} q-1,0\right)$. For $m \equiv 2(\bmod 4)$, let $M^{m}=R P(m-3,0,0,0)$, and if $m \equiv 0(\bmod 4)$, but $m \neq 2^{s}$, let $m=2^{p_{1}}+\cdots+2^{p_{r-1}}+2^{p_{r}}$ with $r>1 p_{1}>\cdots>p_{r-1}>p_{r} \geq 2$ and $M^{m}=$ $R P\left(2^{p_{r}-1}, 2^{p_{r}-1}, 0, m-2^{p_{r}}-3\right)$. This gives indecomposables $M^{m}$ in every dimension not a power of 2 for which $M^{m}$ fibers with fiber $R P(3)$.

Letting $I \subset \Re_{*}$ be the ideal of classes with $\alpha \in I$ if and only if $w_{n}(\alpha)=$ $w_{1}^{n}(\alpha)=0, I$ contains indecomposables $x_{i}$ in dimensions $i$ not of the form $2^{s}$, and if $x_{2 s}=R P\left(2^{s}\right)-R P(2)^{2 s-1}, w_{2 s+1}\left(x_{2 s}^{2}\right)=w_{1}^{2 s+1}\left(x_{2 s}^{2}\right)=0$. If $n=2^{p_{1}}+\cdots$ $+2^{p_{r}}, p_{1}>\cdots>p_{r} \geq 1$, then

$$
\begin{gathered}
w_{n}\left(R P(2)^{n / 2}\right)=1, \quad w_{1}^{n}\left(R P(2)^{n / 2}\right)=0 \\
\left\{\begin{array}{l}
w_{n}\left(R P\left(2^{p_{1}}\right) \times \cdots \times R P\left(2^{p_{r}}\right)\right)=1 \\
w_{1}^{n}\left(R P\left(2^{p_{1}}\right) \times \cdots \times R P\left(2^{p_{r}}\right)\right)=1
\end{array}\right.
\end{gathered}
$$


and so $I$ is the ideal generated by the $x_{i}, i \neq 2^{s}$, and $x_{2}^{2}, s>1$. If $y_{2 s+1} \in I$, $s>1$, are elements of $I$ with $s_{(2 s, 2 s)}\left(y_{2 s+1}\right) \neq 0$, then $y_{2 s+1}=x_{2 s}^{2}$ mod the ideal generated by lower dimensional classes of $I$, and so $I$ is the ideal generated by the $x_{i}, i \neq 2^{s}$, and the classes $y_{2 s+1}$.

Letting $M^{2^{s+1}}=R P\left(2^{s+1}-3,0,0,0\right), M$ fibers over $R P\left(2^{s+1}-3\right)$ with fiber $R P(3)$, and in the notation of Lemma 3.4,

$$
w(M)=(1+a)^{2 s+1-2}(1+c+\alpha)(1+c)^{3}
$$

so $w_{2}(M)=a^{2}+c^{2}+c(c+a)=a^{2}+c \alpha$ and

$$
s_{(2 s, 2 s)}=\left(s_{1,1}\right)^{2 s}=w_{2}^{2 s}=a^{2 s+1}+c^{2 s} \alpha^{2 s}=c^{2 s} \alpha^{2 s} .
$$

Now $c^{4}=c^{3} \alpha$, so $S_{2 s, 2 s}=c^{3} a^{2 s+1}-3$, and $S_{2 s, 2}\left[M^{2 s+1}\right]=1$.

Thus the ideal of classes satisfying $w_{n}(\alpha)=w_{1}^{n}(\alpha)=0$ is the ideal generated by the manifolds $M^{m}$ which fiber with fiber $R P(3)$.

In both Propositions 8.1 and 8.3 , the classes $\alpha \in \Re_{*}$ represented by fiberings with fiber $R P(k), k=2$ or 3 , are precisely the image of the homomorphism $\sigma$ : $\Re_{*}\left(B O_{k+1}\right) \rightarrow \Re_{*}$ assigning to the $(k+1)$-plane bundle $\xi$ over $M$ the cobordism class of $R P(\xi)$. It seems plausible that this could be true for all $k$.

Proposition 8.4. If $R P(k) \stackrel{i}{\rightarrow} M^{n+k} \stackrel{\pi}{\rightarrow} N^{n}$ is a fibering which is totally nonbomologous to zero then $M$ is cobordant to $R P(\xi)$ for some $(k+1)$-plane bundle $\xi$ over an $n$-dimensional manifold.

Proof. Since $\pi$ is totally nonhomologous to zero there is a class $c \epsilon$ $H^{1}\left(M ; Z_{2}\right)$ with $i^{*}(c)=\alpha \in H^{1}\left(R P(k) ; Z_{2}\right)$ and $H^{*}\left(M ; Z_{2}\right)$ is the free $H^{*}\left(N ; Z_{2}\right)$ module on $1, c, \cdots, c^{k}$. Expressing $c^{k+1}$ in this module structure, there are unique classes $a_{1}, \ldots, a_{k+1} \in H^{*}\left(N ; Z_{2}\right)$ with

$$
U=c^{k+1}+\pi^{*}\left(\alpha_{1}\right) c^{k}+\cdots+\pi^{*}\left(\alpha_{r}\right) c^{k+1-r}+\cdots+\pi^{*}\left(\alpha_{k+1}\right)=0 .
$$

Inductively computing $\mathrm{Sq}^{i} U$, and reducing by the relation $U=0$ one obtains universal polynomials $\mathrm{Sq}^{i} \alpha_{j}=\bar{P}_{i, j}\left(\alpha_{1}, \ldots, \alpha_{i+j}\right)$. For example

$$
\begin{aligned}
0=\mathrm{Sq}^{1} U= & \left(\begin{array}{c}
k+1 \\
1
\end{array}\right) c^{k+2}+\left(\begin{array}{l}
k \\
1
\end{array}\right) c^{k+1} \pi^{*}\left(\alpha_{1}\right)+\cdots+\left(\begin{array}{l}
1 \\
1
\end{array}\right) c^{2} \pi^{*}\left(\alpha_{k}\right) \\
& +c^{k} \pi^{*}\left(\mathrm{Sq}^{1} \alpha_{1}\right)+\cdots+c \pi^{*}\left(\mathrm{Sq}^{1} \alpha_{k}\right)+\pi^{*}\left(\mathrm{Sq}^{1} \alpha_{k+1}\right)
\end{aligned}
$$

and 


$$
\begin{aligned}
c^{k+1} \pi^{*}\left(\alpha_{1}\right) & =\pi^{*}\left(\alpha_{1}^{2}\right) c^{k}+\cdots+\pi^{*}\left(\alpha_{1} \alpha_{r}\right) c^{k+1-r}+\cdots+\pi^{*}\left(\alpha_{1} \alpha_{k+1}\right), \\
c^{k+2} & =\pi^{*}\left(\alpha_{1}\right) c^{k+1}+\pi^{*}\left(\alpha_{2}\right) c^{k}+\cdots+\pi^{*}\left(\alpha_{k+1}\right) c \\
& =c^{k}\left(\pi^{*}\left(\alpha_{2}+\alpha_{1}^{2}\right)\right)+\cdots+c\left(\pi^{*}\left(a_{k+1}+\alpha_{k} \alpha_{1}\right)\right)+\pi^{*}\left(\alpha_{k+1} \alpha_{1}\right)
\end{aligned}
$$

and substituting for $c^{k+2}$ and $c^{k+1} \pi^{*}\left(\alpha_{1}\right)$ gives

$$
\mathrm{Sq}^{1} \alpha_{i}= \begin{cases}(i+1) a_{i+1}+\alpha_{i} a_{1}, & i<k+1, \\ a_{k+1} a_{1}, & i=k+1 .\end{cases}
$$

The formulae obtained in this way are precisely the Wu formulae.

Now one may compute the Steenrod operations in the free $H^{*}\left(N ; Z_{2}\right)$ module on $1, c, \cdots, c^{k}$, using the known action on $c$, which is one dimensional, and the relation for $c^{k+1}$, and hence obtain the $W u$ class of $M$ as a universal formula in $c$, the $\alpha_{i}$ and $w_{i}(N)$. By squaring, one obtains the universal formula

$$
w(M)=\pi^{*} w(N)\left\{(1+c)^{k+1}+(1+c)^{k} \pi^{*}\left(\alpha_{1}\right)+\cdots+\pi^{*}\left(\alpha_{k+1}\right)\right\} .
$$

Thus, letting $f: N \rightarrow \Pi_{j=1}^{k+1} K\left(Z_{2}, j\right)$ be the map into a product of EilenbergMac Lane spaces with $f^{*}\left(i_{j}\right)=\alpha_{j}$, there are universal formulae in the characteristic numbers of $f$ which determine the bordism class of $M$.

Since the characteristic numbers of $f$ satisfy all relations given by the $\mathbf{W u}$ formulae, which are the generators for the kernel of the cohomology map $\phi: B O_{k+1}$ $\rightarrow \Pi_{j=1}^{k+1} K\left(Z_{2}, j\right)$ with $\phi^{*}\left(i_{j}\right)=w_{j}$, there is a closed $n$-dimensional manifold $N^{\prime}$ and map $f^{\prime}: N^{\prime} \rightarrow B O_{k+1}$ with $\phi \cdot f^{\prime}: N^{\prime} \rightarrow \Pi_{j=1}^{k+1} K\left(Z_{2}, j\right)$ bordant to $f$ (see [5, (17.3)]).

Letting $\xi$ be the $(k+1)$-plane bundle over $N^{\prime}$ induced by $f^{\prime}, R P(\xi)$ and $M$ have the same Stiefel-Whitney numbers (because of the universal formulae) and so $M$ is bordant to $R P(\xi)$.

Proposition 8.5. The set of classes $a \in \Re_{*}$ which are represented by fiberings with fiber $R P(2 k)$ is precisely the image of $\sigma: \Re_{*}\left(\mathrm{BO}_{2 k+1}\right) \rightarrow \Re_{*}$ assigning to the bundle $\xi$ over $M$ the class of $R P(\xi)$.

Proof. If $R P(2 k) \stackrel{i}{\rightarrow} M^{n} \stackrel{\pi}{\rightarrow} N^{n-2 k}$ is a fibering, $i$ imbeds $R P(2 k)$ in $M$ with trivial normal bundle, so $i^{*}\left(w_{1}(M)\right)=w_{1}(R P(2 k))=\alpha$ and $\pi$ is totally nonhomologous to zero.

In principle, this determines the classes which fiber with fiber $R P(2 k)$, as the ideal generated by the manifolds $R P\left(n_{1}, \ldots, n_{2 k+1}\right)$, but this is far from complete.

There are fiberings with fiber an odd projective space which are not totally nonhomologous to zero; for example the Hopf fibering $R P(1)=S^{1} \rightarrow S^{3} \rightarrow S^{2}$, or 
$R P(3)=\mathrm{SO}_{3} \rightarrow E \rightarrow \tilde{G}$ where $\tilde{G}$ is the Grassmannian of oriented 4 planes in $R^{k}$ and $E$ is the universal $S O(3)$ bundle (I am indebted to E. E. Floyd for this example), and so this method fails in the $R P$ (odd) case.

Turning to more complicated fibers, one has

Proposition 8.6. The set of classes $a \in \Re_{n}$ which are represented by fiberings with fiber $C P(2)$ is precisely the set of classes $\alpha \in \Re_{n}$ for which $w_{1}^{n}(\alpha)=0$.

Proof. If $C P(2) \stackrel{i}{\rightarrow} M^{n} \stackrel{\pi}{\rightarrow} N^{n-4}$ is a fibering, then $i$ is an imbedding with trivial normal bundle, so $i^{*} w_{1}(M)=w_{1}(C P(2))=0$ and $w_{1}(M) \in \operatorname{im} \pi^{*}$. Then $w_{1}^{n}(M)$ $\epsilon \operatorname{im} \pi^{*}$ and is thus zero.

Let $I$ be the ideal in $\Re_{*}$ consisting of classes which fiber with fiber $C P(2)$. Letting $C P\left(n_{1}, n_{2}, n_{3}\right)$ be the complex projective space bundle of $\lambda_{1} \oplus \lambda_{2} \oplus \lambda_{3}$ over $C P\left(n_{1}\right) \times C P\left(n_{2}\right) \times C P\left(n_{3}\right), C P\left(n_{1}, n_{2}, n_{3}\right)$ is bordant to $R P\left(n_{1}, n_{2}, n_{3}\right)^{2}$, and in fact admits a conjugation $c$ with fixed set $R P\left(n_{1}, n_{2}, n_{3}\right)$, and so is bordant as manifold with involution to $R P\left(n_{1}, n_{2}, n_{3}\right)^{2}$ with the twist involution $\tau(x, y)=(y, x)$, as in [5, p. 64]. Taking sequences $\left(n_{1}, n_{2}, n_{3}\right)$ as in Proposition 8.1 , one sees that $l$ contains the square of every class in $\Re_{*}$.

Further, the fibering of $C P\left(n_{1}, n_{2}, n_{3}\right)$ over $C P\left(n_{1}\right) \times C P\left(n_{2}\right) \times C P\left(n_{3}\right)$ is equivariant, both having the obvious conjugation, and so for every manifold $M$, $(M \times M, \tau)$ is bordant as manifold with involution to a manifold $(\bar{M}, c)$ where $\bar{M}$ is a complex manifold with conjugation fibering equivariantly over a manifold with conjugation $(\bar{N}, c)$ and with the fiber being $C P(2)$.

Being given a manifold with involution $(M, t)$, let $P_{m}(M, t)$ be the manifold obtained from $S^{m} \times M$ by identifying $(x, m)$ with $(-x, t(m))$. Define $Q_{m}(M, t)$ as follows. Letting $x=\left(x_{1}, \ldots, x_{m+1}\right) \in S^{m}$ the reflection in the plane $x_{m+1}=0$ induces an involution $T$ on $P_{m}(M, t)$, and $Q_{m}(M, t)$ is obtained from $P_{m}(M, t)$ by identifying $(p, 0)$ with $(T p, 1)$. These are functorial constructions so that if $(\bar{M}, c)$ fibers equivariantly over $(\bar{N}, c)$ with fiber $C P(2)$, then $P_{m}(\bar{M}, c)$ fibers over $P_{m}(\bar{N}, c)$ with fiber $C P(2)$ and $Q_{m}(\bar{M}, c)$ fibers over $Q_{m}(\bar{N}, c)$ with fiber $C P(2)$. Further, the cobordism class of $P_{m}(M, t)$ and $Q_{m}(M, t)$ depend only on the bordism class of $(M, t)$ as manifold with involution.

Letting $(M, t)$ be $(C P(n), c)$ with $c$ the usual conjugation, $P_{m}(M, t)$ is the Dold manifold $P(m, n)$ and $Q_{m}(M, t)$ is the Wall manifold $Q(m, n)$ as defined in [8]. These give indecomposables in $\Re_{*}$ if $m=2^{p}-1, n=2^{p} q$, which give all dimensions $i$ not of the form $2^{s}$ or $2^{s}-1$.

Now $(C P(n), c)$ is bordant to $\left(R P(n)^{2}, \tau\right)$ and this is bordant to $(\bar{M}, c)$ fibering over $(\bar{N}, c)$ with fiber $C P(2)$, and hence there are indecomposables $P_{m}(\bar{M}, c)$ and $Q_{m}(\bar{M}, c)$ which fiber with fiber $C P(2)$ in every dimension not of the form $2^{s}$ or $2^{s}-1$. 
Thus $I$ contains all squares and indecomposables except in dimensions $2^{s}$ and so contains all classes $\alpha \in \Re_{n}$ with $w_{1}^{n}(\alpha)=0$.

One should note that any fibering with fiber $C P(2 k)$ is totally nonhomologous to zero, but one may not be able to choose a class restricting to $\alpha \in H^{2}\left(C P(2 k), Z_{2}\right)$ which has $\mathrm{Sq}^{1} c=0$. This can be done for the projective space bundle of a complex vector bundle, but not for the Dold type manifolds. In particular, the homomorphism $\Re_{*}\left(B U_{3}\right) \rightarrow \Re_{*}$ assigning to $(M, \xi)$ the class of $C P(\xi)$ does not give all c'asses which fiber with fiber $C P(2)$. They can be represented by the $C P(2)$ bundles of real vector bundles of dimension 6 whose structure group is reduced to the group $\widetilde{U}_{3}$ of real linear transformations $T: C^{3} \rightarrow C^{3}$ with $T i= \pm i T$.

This gives partial information about those classes $\alpha \in{ }^{*}$ represented by fiberings with fiber $R P(2)^{2}$. Specifically, the manifolds $(\bar{M}, c)$ used are bordant to involutions $\left(M^{\prime} \times M^{\prime}, \tau\right)$ which fiber eqivariantly over an involution $\left(N^{\prime} \times N^{\prime}, \tau\right)$ with fiber $R P(2)^{2}[5,(24.4)]$. Thus there are indecomposables in all dimensions not of the form $2^{s}, 2^{s}-1$ which fiber with fiber $R P(2)^{2}$. By Proposition 8.1, every decomposable is represented by a fibering with fiber $R P(2)^{2}$. For dimensional reasons no indecomposable of dimension 2 or 4 could fiber with fiber $R P(2)^{2}$, and so one is reduced to

Question. Do there exist indecomposable manifolds $M$ of dimension $2^{s}, s \geq$ 3, which fiber with fiber $R P(2) \times R P(2)$ ?

9. Manifolds with involution. Our results may be applied to manifolds with involution, and in this section, we will make this application.

Following Conner and Floyd [5], if $M^{n}$ is a closed manifold with differentiable involution $T$, then the fixed set of $T$ is the disjoint union of closed submanifolds $F^{k}$, and if $\nu_{n-k}$ is the normal bundle of $F^{k}$ in $M^{n}$ then the manifold $M$ is bordant to $\bigcup_{k} R P\left(\nu_{n-k} \oplus \underline{1}\right)$ and the bordism class in $\eta_{n-1}\left(B O_{1}\right)$ given by the sum of the classes $\left[R P\left(\nu_{n-k}\right), \lambda\right]$ is zero.

The question to be considered here is:

Which classes $a \in \eta_{n}$ can be represented by manifolds $M^{n}$ having a differentiable involution $T$ for which the fixed set of $T$ is a submanifold $F$ of dimension $k$ ?

When $k=n$, every class can be so represented, since every manifold $M$ has the trivial involution $T(x)=x$. When $k=n-1, M$ must bound for $\left[R P\left(\nu_{1}\right), \lambda\right]=$ $\left[F^{n-1}, \nu_{1}\right]$ bounds and so $\left[R P\left(\nu_{1} \oplus \underline{1}\right)\right]=0$.

Proposition 9.1. If $M^{n}$ is a differentiable manifold with involution $T$ with fixed set a submanifold $F$ of dimension $k<n-1$, then $w_{1}^{n}[M]=0$.

Proof. Let $F^{k}$ be the fixed set of $T$ with normal bundle $\nu_{n-k}=\nu$. Then $M$ is bordant to $R P(\nu \oplus \underline{1})$ which fibers over $F$ with fiber $R P(n-k)$. If $n-k$ is odd, then by Proposition 8.2, $w_{1}^{n}[M]=0$, and so one may assume $n-k$ is even. Using the notation of Lemma 3.4, the Stiefel-Whitney class of $R P(\nu \oplus \underline{1})$ is 
so

$$
w(F) \cdot\left\{(1+c)^{n-k+1}+(1+c)^{n-k} w_{1}(\nu)+\cdots+(1+c) w_{n-k}(\nu)\right\}
$$

$$
w_{1}(R P(\nu \oplus \underline{1}))=w_{1}(F)+c+w_{1}(\nu)
$$

and

$$
\begin{aligned}
w_{1}^{n}(R P(\nu \oplus \underline{1}))= & \left(c+w_{1}(F)+w_{1}(\nu)\right)^{n}=\sum_{j=0}^{n}\left(\begin{array}{l}
n \\
i
\end{array}\right) c^{n-i}\left(w_{1}(F)+w_{1}(\nu)\right)^{i} \\
& =\sum_{i=0}^{k}\left(\begin{array}{l}
n \\
i
\end{array}\right) c^{n-i}\left(w_{1}(F)+w_{1}(\nu)\right)^{i}=\sum_{i=0}^{k}\left(\begin{array}{l}
n \\
i
\end{array}\right) c^{n-k} \bar{w}_{k-i}(\nu)\left(w_{1}(F)+w_{1}(\nu)\right)^{i}
\end{aligned}
$$

and so

$$
w_{1}^{n}[M]=\sum_{i=0}^{k}\left(\begin{array}{l}
n \\
i
\end{array}\right) \bar{w}_{k-i}(\nu)\left(w_{1}(F)+w_{1}(\nu)\right)^{i}[F] .
$$

On the other hand, $(R P(\nu), \lambda)$ bounds in $\Re_{*}\left(B O_{1}\right)$ and

$$
w(R P(\nu))=w(F) \cdot\left\{(1+c)^{n-k}+(1+c)^{n-k-1} w_{1}(\nu)+\cdots\right\}
$$

so

$$
w_{1}(R P(\nu))=w_{1}(F)+w_{1}(\nu)
$$

and for $0 \leq i \leq k$

$$
\begin{aligned}
0 & =w_{1}(\lambda)^{n-i-1} \cdot w_{1}(R P(\nu))^{i}[R P(\nu)] \\
& =c^{n-i-1} \cdot\left(w_{1}(F)+w_{1}(\nu)\right)^{i}[R P(\nu)] \\
& =c^{n-k-1} \bar{w}_{k-i}(\nu) \cdot\left(w_{1}(F)+w_{1}(\nu)\right)^{i}[R P(\nu)] \\
& =\bar{w}_{k-i}(\nu) \cdot\left(w_{1}(F)+w_{1}(\nu)\right)^{i}[F] .
\end{aligned}
$$

Combining these, $w_{1}^{n}[M]=0$.

Proposition 9.2. A class $a \in \Re_{n}$ is represented by a manifold $M^{n}$ with differentiable involution $T$ with fixed set $F$ of dimension $n-2$ if and only if $w_{1}^{n}(\alpha)=0$.

Proof. The set of classes $a \in \Re_{*}$ with this property is an ideal $J$ for if $T$ : $M^{n} \rightarrow M^{n}$ fixes $F^{n-2}$, then $1 \times T: P \times M \rightarrow P \times M$ fixes $P \times F$, and by Proposition 9.1 this is contained in the ideal $I$ of classes $a \in \Re_{n}$ with $w_{1}^{n}(a)=0$.

By Proposition 8.3, the ideal $I$ is generated by classes $R P\left(n_{1}, n_{2}, n_{3}, n_{4}\right)$ and the class of $R P(2)^{2}$. If $\xi, \eta$ are 2-plane bundles over $M$, then $R P(\xi \oplus \eta)$ has an involution $T$ induced by multiplication by -1 in the fibers of $\xi$ and 1 
in the fibers of $\eta$, and the fixed set of $T$ is $R P(\xi) \cup R P(\eta)$, which has codimension 2. Thus $\left[R P\left(n_{1}, n_{2}, n_{3}, n_{4}\right)\right] \in J$. The involution $\tau: R P(2) \times R P(2) \rightarrow R P(2) \times$ $R P(2):(x, y) \rightarrow(y, x)$ fixes the diagonal $R P(2)$, so $R P(2)^{2} \in J$. Thus $I \subset J$ and so $I=J$.

Since any class in $\Re_{*}\left(\mathrm{BO}_{2 k}\right)$ is represented by a manifold $M$ and the Whitney sum of two $k$ plane bundles, the above construction shows that every class in the image of $\sigma: \Re_{i}\left(B_{2 k}\right) \rightarrow \Re_{i+2 k-1}$ is represented by a manifold with involution with fixed set of codimension $k$.

It is curious that the ideal of classes $\alpha \in \Re_{n}$ with $w_{1}^{n}(\alpha)=0$ is the ideal generated by Wall's ${ }^{2}{ }_{*}$ consisting of those classes for which all numbers divisible by $w_{1}^{2}$ are zero.

\section{REFERENCES}

1. R. L. W. Brown, Cobordism and bundles over spheres, Michigan Math. J. 16 (1969), 315-320. MR $40 \# 3558$.

2. R. O. Burdick, Oriented manifolds fibered over the circle, Proc. Amer. Math. Soc. 17 (1966), 449-452. MR $36 \# 3368$.

3. P. E. Conner, The bordism class of a bundle space, Michigan Math. J. 14(1967), 289-303. MR $37 \# 3579$.

4. P. E. Conner and E. E. Floyd, Fibring within a cobordism class, Michigan Math. J. 12 (1965), 33-47. MR 31 \#4038.

5. - Differentiable periodic maps, Springer-Verlag, Berlin, 1964. MR $31 \# 750$.

6. W. S. Massey, Algebraic topology: An introduction, Harcourt, Brace and World, New York, 1967. MR 35 \#2271.

7. J. W. Milnor, A procedure for killing homotopy groups of differentiable manifolds, Proc. Sympos. Pure Math., vol. 3, Amer. Math. Soc., Providence, R. I., 1961, pp. 39-55. MR 24 \#A556.

8. C. T. C. Wall, Determination of the cobordism ring, Ann. of Math. (2) 72 (1960), 292-311. MR $22 \# 11403$.

DEPARTMENT OF MATHEMATICS, UNIVERSITY OF VIRGINIA, CHARLOTTESVILLE, VIRGINIA 22903 